\title{
Hacia la profesionalización de la pedagogía hospitalaria
}

\author{
María Cruz Molina Garuz ${ }^{1}$
}

\section{RESUMEN}

Se presentan las bases teóricas y las orientaciones prácticas, con el objetivo de contribuir al avance de la profesionalización en la educación hospitalaria. Se enmarcan en un concepto amplio de pedagogía hospitalaria, cuyos ámbitos de actuación se conciben como parte de un sistema educativo inclusivo de calidad. Se analizan los conceptos de profesionalidad y de desarrollo profesional del docente para su aplicación al contexto hospitalario, como elementos clave en la definición de su identidad propia. Se define el perfil competencial de esta profesión mediante la delimitación de funciones y competencias, aportando a modo de ejemplo el desarrollo de la competencia responsabilidad. Este marco puede servir de base para la formación de profesionales, que a su vez será clave para el desempeño competente de la profesión. Derivado de este análisis, se hace énfasis en la importancia de la formación desde un enfoque de enseñanza y aprendizaje basado en las competencias profesionales.

PALABRAS-CLAVE: Pedagogía hospitalaria. Profesionalización y desarrollo profesional. Formación basada en competencias. Docente. Educación hospitalaria.

\section{Rumo à profissionalização da pedagogia hospitalar}

\section{RESUMO}

Este artigo tem com o objetivo de contribuir com o avanço da profissionalização na educação em hospitais, segundo o conceito da Pedagogia Hospitalar cujo campo de atuação é concebido como parte de um sistema inclusivo de qualidade. Serão analisados os conceitos de

\footnotetext{
${ }^{1}$ Doctora em Medicina. Universidad de Barcelona. España. Orcid: https://orcid.org/0000-0002-1600-2938. E-mail: cmolina@ub.edu.
} 
profissionalidade e de desenvolvimento profissional, como elementos fundamentais para definir a identidade própria deste profissional. Define-se o perfil de competências para esta modalidade profissional, mediante a delimitação de funções e competências, tendo como exemplo o desenvolvimento da competência responsabilidade. Esta abordagem pode ser considerada como base para a formação de profissionais que por sua vez serão essenciais para o desempenho competente da profissão. Advinda desta análise, se enfatiza a importância da formação a partir da abordagem de ensino-aprendizagem baseada em competências profissionais.

PALAVRAS-CHAVE: Pedagogia hospitalar. Profissionalização e desenvolvimento profissional. Formação baseada em competências. Docente. Educação hospitalar.

\title{
Towards the professionalization of hospital pedagogy
}

\begin{abstract}
In this article, we present theoretical bases and practical orientations, with the objective of contributing to the advancement of professionalisation of Hospital Education. We frame it in a wide concept of Hospital Pedagogy, which areas of action are conceived as a part of an inclusive and high-quality educational system. We analyse the concepts of professionality and the education professionals' professional development, for its application in the hospital context, as key elements to define its own identity. We define the competencies profile of this profession through the functions and competencies delimitation, giving as an example the development of responsibility competency. This frame can be used as a base for professionals' training and, at the same time, be a key for the competent exercise of the profession. From this analysis, we emphasise the importance of training from a teaching-learning approach based on professional competencies.
\end{abstract}

KEYWORDS: Hospital Pedagogy. Professionalisation and professional development. Training based in competencies. Teaching. Hospital Education.

$$
* * *
$$




\section{Introducción}

La calidad de la atención educativa, sanitaria y social viene determinada en gran parte por las características de la práctica profesional que dependen de las cualidades, actitudes y aptitudes de los profesionales; del contexto institucional, así como de su reconocimiento social.

El docente hospitalario (DH) debe estar capacitado para desarrollar sus funciones con la máxima competencia y garantizar el derecho a una educación de calidad en cualquiera de los contextos en que tiene que actuar, para lo cual requiere una especialización.

La educación hospitalaria cumple a su vez la misión de reducir el impacto biopsicosocial que representa enfrentarse a una situación de vulnerabilidad imprevista y que genera mucha incertidumbre y miedo. La hospitalización origina una serie de circunstancias de riesgo a las que se debe dar apoyo desde los espacios educativos (ORTIZ, 1999): sentirse en un lugar extraño con personas desconocidas; separación de la familia; ruptura con la vida cotidiana y la escuela; incertidumbre; mayor pasividad aunque se disponga de más tiempo libre; malestar físico y emocional; preocupación por los proyectos de futuro. En este contexto se articulan 5 focos de atención: el sanitario, el psicológico, el educativo, el social, cuyos profesionales deben coordinarse y colaborar en un enfoque de atención integrada, centrada en la persona, donde el niño, la niña, el adolescente y su familia son el eje del sistema, tal como lo plantea la Conferencia internacional sobre atención integrada (AGENCIA DE CALIDAD Y EVALUACIÓN SANITARIAS DE CATALUNYA, 2016), incidiendo en el trabajo colaborativo y en la multidisciplinariedad.

\section{Un marco conceptual para la educación en situación de enfermedad}

La educación dirigida a población infantil y juvenil que presenta algún problema de salud se emmarca en la tradicional pedagogía 
hospitalaria. Esta denominación, aunque no se corresponde estrictamente con la evolución que ha desarrollado esta disciplina, tanto por la población destinataria, como por los ámbitos de intervención, le otorga identidad a la profesión. En un intento de ofrecer un concepto amplio y actualizado, la definimos como: "Una disciplina de carácter científico, académico y profesional que estudia e integra actuaciones educativas y psicoeducativas de calidad dirigidas a las personas con problemas de salud y a sus familias, con el objeto de garantizar el cumplimiento de sus derechos, dar respuesta a las necesidades biopsicosociales, desarrollar sus potencialidades y mejorar la calidad de vida" (MOLINA, 2020). La planteamos a lo largo de la vida ya que la enfermedad puede aparecer en cualquier momento vital y cuando se presenta, puede durar largos períodos de tiempo o cronificarse. Enfatizamos la atención centrada en la persona y en la familia con el fin de mejorar la calidad de vida y potenciar las capacidades, lo cual lleva implícito un enfoque resiliente. Por otro lado, los contextos de actuación se ubican tanto en espacios hospitalarios mediante los servicios educativos dentro y fuera del aula o escuela, como fuera del hospital ya sea el domicilio, una casa de acogida o la escuela regular, puesto que en cualquier contexto educativo, una de las situaciones de diversidad en el alumnado puede ser una enfermedad. Podríamos decir que la acción educativa debe desarrollarse en todos los contextos donde se encuentra la persona con enfermedad (MOLINA; BORI, 2013). Por otro lado proponemos que el servicio educativo hospitalario, así como el que se ofrece en otros contextos forme parte de un sistema educativo inclusivo (Fig. 1). 
FIGURA 1: La pedagogía hospitalaria parte del sistema educativo inclusivo

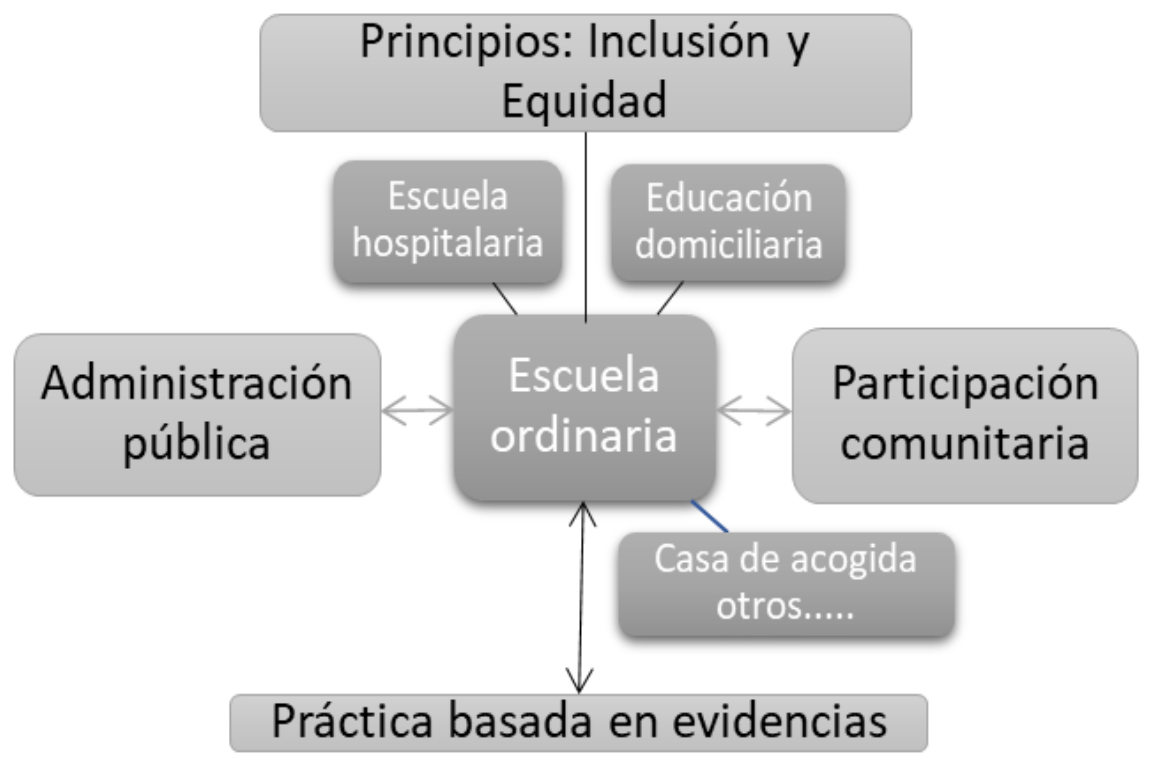

Fuente: Adaptado de Ainscow (2020)

\section{Profesionalidad y desarrollo profesional del docente hospitalario}

Las condiciones que se requieren para la buena práctica profesional del docente hospitalario son diversas, por lo cual conviene delimitarlas. Destacaremos la profesionalidad y el desarrollo profesional.

La profesionalidad implica contemplar la especificidad de la profesión, que en el caso del docente es "el conjunto de comportamientos, conocimientos, destrezas, actitudes y valores que constituyen la especificidad de ser profesor" (SACRISTÁN, 1991, citado por OLIVEIRA, 2013), a la vez que conforma la identidad de la profesión (OJEDA, 2008). Implica compromiso con la profesión y ser competente, es decir actuar con eficacia. Tejada (2002), distingue entre profesionalidad tradicional, en la que el profesional es un mero transmisor de conocimientos y se basa en una racionalidad técnica y profesionalidad alternativa o profesionalidad desarrollada, en la que la profesión es dinámica y sujeta a los cambios. El profesional aplica la reflexión de su práctica y adquiere un rol de facilitador del aprendizaje. Desde este enfoque la propia práctica 
profesional es un escenario de aprendizaje y los procesos de enseñanza y aprendizaje son "democráticos, dialógicos y ecológicos, dentro de contextos institucionales orientados a la mejora y al crecimiento organización que aprende y se desarrolla" (TEJADA, 2002, p. 65).

En el caso del o la docente hospitalaria la especificidad viene definida fundamentalmente por cuatro factores: 1) el entorno donde desempeña su profesión (sanitario y domiciliario, entre otros), 2) las funciones que realiza además de las docentes, 3) la metodología que aplica y, 4) las características de la población que atiende. Otros elementos a considerar cada vez más identitarios de esta profesión, son: la vinculación con la escuela regular (coordinación, trabajo colaborativo) y con los profesionales de la salud, de la psicología y del trabajo social; el abordaje de las temáticas escolares de acuerdo al currículum de la etapa; la atención personalizada por la diversidad del alumnado atendido (además de las que son habituales, el estado de salud y emocional); el apoyo y acompañamiento en la gestión emocional de las familias, entre otros (MOLINA, 2020). El enfoque de la profesionalidad desarrollada, es pertinente en pedagogía hospitalaria, para ofrecer una educación de calidad. La acción en el aula hospitalaria es dinámica y cambiante, por lo que requiere una continua adaptación y flexibilidad.

La profesionalidad del docente hospitalario implica la adquisición de capacidades y habilidades que le permitan integrar la reflexión sobre su práctica y contrastar los fundamentos teóricos y metodológicos con la propia experiencia tanto a nivel individual como colectivo, de manera que sea una forma de desarrollo profesional. Por otro lado el trabajo colaborativo en contextos favorecedores, tal como afirma Tejada (2002), es el que produce un verdadero desarrollo profesional.

El desarrollo profesional del docente se entiende como un "grupo de factores que hacen posible $u$ obstaculizan el avance de los profesores en su vida profesional" (IMBERNÓN; CANTO, 2013, p. 2). En sentido restringido se refiere a la formación como único factor, pero en un sentido más amplio del concepto, se conjuga la formación con otros factores no menos importantes 
que influyen de manera significativa, como las condiciones de trabajo, las posibilidades de promoción y el reconocimiento social de la profesión, entre otros. La profesión de docente hospitalario requiere condiciones de trabajo dignas, pero especialmente el conocimiento y reconocimiento social, pues se trata de una profesión de gran compromiso que aporta grandes beneficios a la sociedad. El propio docente debe tomar conciencia de ello y ser proactivo en la defensa de su identidad como profesional.

Imbernón y Canto (2013) consideran que la formación puede contribuir al desarrollo profesional; sin embargo la falta de otros factores, como un salario adecuado, puede incidir negativamente y precarizar la profesión. Esta visión más amplia del concepto de desarrollo profesional, debe entenderse como "todo intento sistemático de mejorar la práctica laboral, creencias y conocimientos profesionales, con el propósito de aumentar la calidad docente, investigadora y de gestión”. La educación de calidad en situación de enfermedad debe ser la meta hacia donde debe avanzar el profesional de la PH. Así pues, para Imbernón y Canto (2013. pp.4-5) son cinco los ejes de actuación para el desarrollo profesional del docente, que podemos aplicar al hospitalario:

1. La reflexión prácticoteórica sobre la propia práctica mediante el análisis de la realidad; 2. El intercambio de experiencias entre iguales para posibilitar la actualización en todos los campos de intervención educativa; 3. La unión de la formación a un proyecto de trabajo; 4. La formación como análisis crítico a prácticas laborales, como la jerarquía, el sexismo, la proletarización, el individualismo, el bajo estatus, entre otras; y 5 . El desarrollo profesional que se da predominantemente en el centro educativo.

El trabajo en red de los docentes hospitalarios es otro de los retos a alcanzar para conseguir el intercambio de experiencias que conducirá a la construcción conjunta de buenas prácticas educativas en contextos sanitarios.

Aunque existe abundante bibliografía respecto al desarrollo profesional y profesionalidad del docente de la escuela regular, no se puede afirmar lo mismo en relación con el docente hospitalario. Es por 
ello que resulta necesario delimitar y definir esta profesión de modo que se le otorgue identidad propia y sea reconocida socialmente por su rol para garantizar el derecho a la educación de calidad de las personas con problemas de salud. Para ello, es necesario dotar a esa profesión de las condiciones necesarias que la dignifiquen y la situen en el lugar que le corresponde, a saber: como parte del sistema educativo inclusivo (MOLINA, 2020). Tal como afirman Albertoni y Chiari (2014), los órganos públicos deben establecer lazos entre sí para configurar una red de apoyo a la educación hospitalaria.

Uno de los factores más relevantes que requiere el desarrollo profesional, aunque como hemos visto no el único, es la formación. Ésta debe estar enfocada al reconocimiento del carácter profesional específico y a la valoración social de la misma, empezando por el propio profesional que se debe considerar un agente de cambio social. Del mismo modo debe contribuir a la construción de la pedagogía hospitalaria, como disciplina y como profesión comprometida con la sociedad.

\section{Perfil competencial del docente hospitalario}

El perfil del docente hospitalario se construye a partir de la adquisición de las competencias necesarias para el desarrollo de sus funciones docentes y del acompañamiento en el proceso de enseñanza y aprendizaje. Para ello se requiere una sólida formación, que junto a la experiencia profesional, la actitud abierta al conocimiento, la responsabilidad y el compromiso configuran la profesión del docente hospitalario. Para Bozu (2007, p.4), “definir el perfil profesional del profesorado significa definir las funciones, las atribuciones, los ámbitos de actuación y sus competencias profesionales". Respecto a las atribuciones personales González-Simancas y Polaino-Lorente (1990), consideran que ha de ser un profesional imaginativo, exigente pero a la vez flexible, priorizando la salud ante los aprendizajes y el currículum. 


\section{Funciones del docente hospitalario}

Según diversas autoras (CASTILLO, 2015; GENERALITAT DE CATALUNYA, 2007; GRAU; ORTIZ, 2001; LIZASOÁIN, 2016; ORTIZ, 1999) podemos agrupar las funciones del docente hospitalario en:

- Educativa y psicoeducativa. Es la función que le da sentido a la profesión de docente hospitalario. Desde sus orígenes, la educación, siguiendo los objetivos y contenidos curriculares, ha sido la función primordial. Ha devuelto el rol de alumno o alumna al niño, niña o adolescente hospitalizado, sustituyendo al de paciente. Por otro lado, ha contribuido a reducir el impacto negativo de la hospitalización, dada la importancia de seguir el proceso educativo iniciado en la escuela ordinaria, respetando los ritmos y adecuando los procesos según la diversidad curricular. En la actualidad, la educación se enfoca de forma integral, y el docente ejerce un rol de acompañamiento educativo para la adquisición de habilidades cognitivas, emocionales y sociales. En situación de enfermedad este rol va dirigido también a la adquisición de estrategias para el afrontamiento resiliente de las situaciones adversas y preparar para la comprensión de la enfermedad y la hospitalización. Para Albertoni y Chiari (2014), el docente hospitalario es un profesional que acompaña en el proceso de aprendizaje para una mejor calidad de vida social y personal del alumnado.

- Acompañamiento familiar. Los padres y madres deben formar parte activa en los procesos asistenciales del hijo o hija, siendo a su vez población destinataria desde el modelo de atención centrado en la familia. Éste debe contemplar también a los hermanos y hermanas y otros miembros próximos de la familia. El y la docente hospitalaria pueden orientar a las familias en la identificación de necesidades (básicas, de cuidado, educativas, emocionales y afectivas), en los procesos educativos y en la forma de relacionarse con los hijos e hijas, así como en la autogestión emocional y el afrontamiento resiliente. 
- Innovación: la introducción de prácticas innovadoras incidirán en la mejora y efectividad de los resultados. Es considerado un factor de calidad de la acción educativa.

- Coordinación: para una docencia efectiva el docente hospitalario debe trabajar de forma colaborativa con los docentes domiciliarios, con los de la escuela regular y con los equipos psicopedagógicos. Así mismo es necesaria la coordinación con el equipo sanitario, compartiendo información y tomando decisiones conjuntas en áreas en las que se requiera la transdisciplinariedad.

- Gestión: Los docentes pueden ejercer la función de dirección del centro educativo, asumiendo funciones de tipo organizativo, administrativo, de supervisión, de coordinación, de orientación a las familias sobre los procesos, gestión económica y realización de informes, memorias, planificación entre otras.

- Investigación: La acción profesional y la investigación son dos funciones que se complementan y que se necesitan entre sí. La pedagogía hospitalaria basada evidencias y en buenas prácticas requiere conocer y participar en investigaciones relacionadas con el ámbito. El enfoque participativo de los estudios de investigación es el más pertinente, puesto que recoge las voces de las personas implicadas en la actuación educativa y permite construir un cuerpo de conocimientos que le darán rigor y valor a la pedagogía hospitalaria.

-Autocuidado: se basa en las acciones que derivan de la previa identificación de factores de riesgo y de protección tanto personales como del contexto hospitalario, para mejor la satisfacción con el trabajo y la prevención de burnout.

-Aplicación de los principios éticos: La ética y la bioética deben aplicarse en todas las acciones educativas, psicoeducativas, de investigación, así como en las relaciones con otros profesionales. 


\title{
Competencias profesionales para la educación hospitalaria
}

Partimos de la complejidad del propio concepto de competencia, del que se han formulado múltiples definiciones. Rodriguez, Serreri y Del Cimuto (2010: p. 35-36), refieren los siguientes aspectos comunes a todas ellas:

\begin{abstract}
a) Denotan un saber hacer, de una correcta integración entre habilidades y conocimientos o saberes; b) Demuestran que ese saber hacer no es algo simple y lineal sino un modo de proceder complejo; c) También demuestran que esa complejidad cubre un amplio espectro de habilidades, a saber: las cognitivas, las afectivas, las sociales o las psicomotrices; d) La competencia para saber hacer, es decir para saber poner en marcha una acción, se refiere a un conjunto de situaciones o contextos concretos y no forzosamente a cualquier situación (competencia situada).
\end{abstract}

Haciendo énfasis en la competencia como un proceso integrador, la definen como "un saber hacer complejo resultado de un proceso de integración, de movilización y de gestión de capacidades, destrezas y conocimientos usados de maneras eficaces y en situaciones con un hilo conductor común".

Esta perspectiva conceptual resulta de especial interés para su aplicación en la educación hospitalaria. Implica que el profesional docente para ser competente ha de poseer un conjunto de capacidades profesionales necesarias para el desarrollo efectivo de las funciones que les son propias. Estas funciones, en su conjunto, requieren un abordaje multidimensional que integre habilidades cognitivas, emocionales y sociales, en un contexto específico y distinto a la función docente regular, como es el hospitalario.

Siguiendo a los mismos autores (RODRÍGUEZ MORENO; SERRERI; DEL CIMMUTO, 2010. p. 37), es importante desglosar cada competencia en capacidades y destrezas, siendo la capacidad "agrupamiento de destrezas", o "elemento constitutivo de la competencia". La destreza sería "un saber-hacer simple que integra los conocimientos declarativos", es decir los saberes. Así podemos considerar que la adquisición de competencias laborales es un proceso complejo (fig 2.) que 
requiere estrategias de enseñanza y aprendizaje, innovadoras y efectivas, como son la metolodología grupal, la experiencial y la cooperativa.

FIGURA 2: Proceso de desarrollo de la competencia

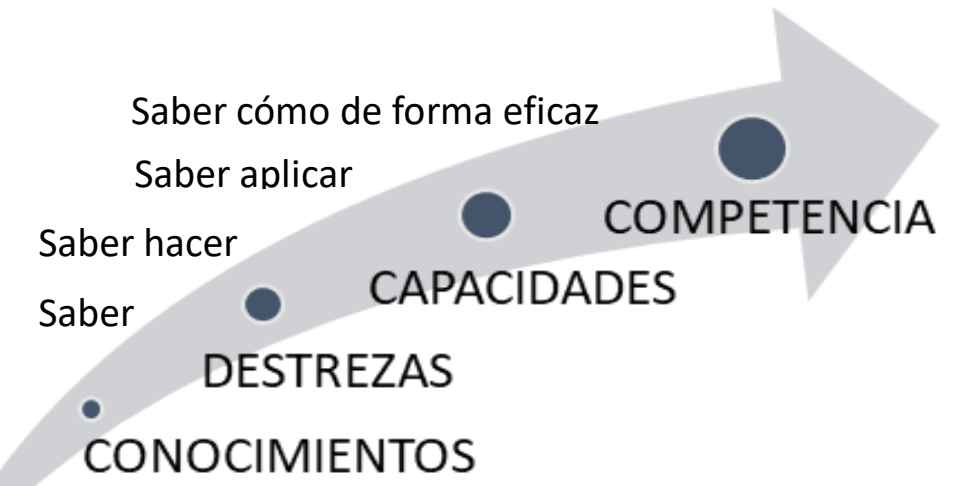

Fuente: Elaboración propia

El desarrollo de competencias profesionales del docente hospitalario se produce combinando procesos formativos continuados, experiencia profesional y práctica reflexiva, del mismo modo que en otros ámbitos profesionales. La consideración de la pedagogía hospitalaria como profesión desde una perspectiva de calidad educativa, requiere la definición y revisión continua del perfil de competencias específico en base a las funciones que desarrolla, a la investigación y a la formación básica, especializada y continuada. Para ello se deberían integrar en las organizaciones responsables, los correspondientes procesos evaluativos y autoevaluativos aplicando estrategias como por ejemplo el balance de competencias propuesto por Rodríguez Moreno (2006), muy útil para la autoidentificación. De este modo cada profesional toma conciencia y puede aplicar mejoras respecto a su proyecto profesional.

Para el desarrollo de las funciones descritas en el apartado anterior es preciso concretar las tareas que comporta cada una de ellas. La delimitación de las competencias vinculadas a cada tarea, según Rodríguez Moreno 
(2006, p.71), requiere comprender cómo el profesional “activa su bagage competencial" refriéndose a la forma en que resuelve los problemas, los medios utilizados y las estrategias que aplica para conseguir los objetivos. Así, esta autora entiende la competencia profesional como la "capacidad general que una persona posee, aunque ligada a una tarea laboral concreta y que exige la interpretación del contexto, el conocimiento de las normas y la implementación de soluciones eficaces”. En pedagogía hospitalaria reiteramos la particularidad del desempeño docente en un entorno complejo como es el hospital, en el que el profesional debe conjugar las normas que afectan al proceso de enseñanza y aprendizaje en distintos espacios de actuación, con las que rigen en el hospital, incluyendo las de higiene y seguridad de toda institución sanitaria. Por otro lado, las soluciones a las que tienen que dar respuesta el y la docente hospitalarios son diversas y de mayor complejidad que en la docencia ordinaria. Los objetivos van más allá de la enseñanza curricular, necesariamente diversificada, para conseguir mejoras en el bienestar y la calidad de vida, y también en el nivel de satisfacción con las atenciones recibidas. De las distintas perspectivas que se han aportado respecto a la competencia profesional, deducimos que la que mejor se adecúa al ámbito que nos ocupa es la entendida como participación en una comunidad de práctica profesional, puesto que contempla la experiencia adquirida como metodología constructiva, base de una "mentalidad heurística, investigadora, núcleo central de una mentalidad competente" (RODRÍGUEZ MORENO, 2006, p.75). En pedagogía hospitalaria, gran parte del saber se ha generado desde la experiencia profesional, puesto que la construcción del conocimiento desde la investigación es un proceso relativamente reciente y posterior al origen de las primeras acciones pedagógicas en los hospitales (MOLINA, 2020). Es preciso integrar en la práctica profesional esa mentalidad heurística, es decir orientada a indagar y descubrir formas de comprender y analizar las situaciones, creativas y simplificadoras, que ayuden a tomar decisiones y a resolver problemas. Otros componentes de este enfoque son el desarrollo de 
la profesionalidad colectiva, la cooperación y la autoregulación. El trabajo del y la maestra hospitalarios en algunos casos es individual y pertenece al día a día laboral, pero en su conjunto ha contribuido a la construcción de la identidad profesional del docente hospitalario, favorecido por los foros de difusión, el debate y las tecnologías, junto a las redes nacionales e internacionales que trabajan proactivamente para que la educación hospitalaria llegue a todas las personas sin excepción.

La competencia profesional debe contemplarse desde su tridimensionalidad, tal como sugiere LeBoterf: dimensión subjetiva (biografía y competencias sociales); dimensión laboral (situación profesional); y, dimensión formativa (cualificación). La competencia sería la zona de intersección entre ellas (RODRÍGUEZ MORENO, 2006).

Las competencias del docente hospitalario deben integrar las del docente de la escuela ordinaria con las específicas de la educación hospitalaria. La metodología del Proyecto Tuning (Tuning educational estructures in Europe) (COMITÉ DE GESTIÓN DE TUNING, 2006) plantea dos tipos de competencias profesionales: las genéricas y las específicas.

Las competencias genéricas (transversales) las clasifica en instrumentales (cognitivas, metodológicas, tecnológicas y linguísticas), interpersonales (capacidades individuales $\mathrm{y}$ habilidades sociales), y sistémicas (comprensión, sensibilidad y conocimientos). Las específicas serían las correspondientes a cada área. A su vez, la Agencia Nacional de Evaluación de la calidad y la acreditación (ANECA), ha propuesto otro modelo de clasificación para las competencias genéricas (RODRÍGUEZ MORENO, 2006, p. 181): saber (poseer conocimientos), saber hacer (dominar los métodos), saber aprender (ser capaz de formarse), saber estar (mostrar actitudes y formas de actuar) y hacer saber (posibilitar el desarrollo de aprendizajes desde la intercomunicación personal)2 .

\footnotetext{
${ }^{2}$ Ver también: Rodríguez-Moreno, M,.L. (2014). La pasión por aprender a aprender. Desarrollo de la competencia estratégica. Guía didáctica para la universidad y la empresa. Barcelona: Laertes.
} 
Para el profesional de la pedagogía hospitalaria, tomando como base las competencias transversales (genéricas) de la Universidad de Barcelona (UNIVERSITAT DE BARCELONA, 2008) proponemos las siguientes:

Compromiso ético. Comportamiento consecuente con los valores personales y el código deontológico. Aceptación de los estándares éticos de la profesión. Comprometerse con la identidad y ética profesional. Reconocimiento y respeto de las diversidades.

Capacidad de aprendizaje, autonomía y responsabilidad. Capacidad de análisis y de síntesis, de aplicación de la teoría a la práctica. Disposición para implicarse en el trabajo de manera eficiente y autónoma.

Trabajo en equipo. Relacionarse y colaborar en la realización de tareas dentro de un equipo interdisciplinar y multicultural y desde una perspectiva compleja. Contribuir a un proyecto común.

Capacidad comunicativa. Saber expresar las ideas de forma clara, así como escuchar y entender las ideas de los otros en diferentes situaciones. Saber buscar e integrar la información.

Resolución de problemas y toma de decisiones. Identificar, analizar y definir los elementos significativos que constituyen un problema para resolverlo de forma efectiva con criterio.

Adaptabilidad. Adaptación a las situaciones cambiantes, actuando con versatilidad y flexibilidad teniendo en cuenta el estado emocional y de salud de las personas.

Sostenibilidad. Saber valorar el impacto social y medioambiental de las actuaciones en el ámbito. Capacidad de manifestar visiones integradas y sistémicas.

Capacidad creativa y emprendedora. Capacidad de formular, diseñar y gestionar proyectos. Capacidad de buscar e integrar nuevos conocimientos y actitudes.

Respecto a las competencias específicas, en base al modelo de ANECA (RODRÍGUEZ MORENO, 2006), mostramos las más significativas (tabla 1). 
TABLA 1: Competencias específicas del docente hospitalario

\begin{tabular}{|c|c|}
\hline \multirow{5}{*}{$\begin{array}{c}\text { Saber } \\
\text { (conocimientos) }\end{array}$} & Conocer las bases teóricas y epistemológicas de la pedagogía hospitalaria. \\
\hline & $\begin{array}{l}\text { Reconocer la atención psicoeducativa de personas con enfermedad } \\
\text { desde una mirada transdisciplinar, inclusiva y holística. }\end{array}$ \\
\hline & $\begin{array}{l}\text { Comprender las teorías y modelos de atención psicoeducativa a lo } \\
\text { largo de la vida. }\end{array}$ \\
\hline & $\begin{array}{l}\text { Integrar conocimientos adquiridos teóricos, metodológicos, aplicados en } \\
\text { diferentes campos de estudio de la pedagogía hospitalaria. }\end{array}$ \\
\hline & $\begin{array}{l}\text { Conocimiento de los métodos y las estrategias de la investigación } \\
\text { aplicada a la pedagogía hospitalaria. }\end{array}$ \\
\hline \multirow{11}{*}{$\begin{array}{l}\text { Saber hacer } \\
\text { (destrezas / } \\
\text { habilidades) }\end{array}$} & Gestión de la convivencia \\
\hline & Gestión organizativa y económica \\
\hline & $\begin{array}{l}\text { Análizar las necesidades psicoeducativas de las personas con } \\
\text { problemas de salud y sus familias. }\end{array}$ \\
\hline & $\begin{array}{l}\text { Desarrollar propuestas de intervención, basadas en la evidencia } \\
\text { científica y en las buenas prácticas según la etapa vital y el contexto. }\end{array}$ \\
\hline & $\begin{array}{l}\text { Identificar y aplicar estrategias didácticas efectivas según la etapa } \\
\text { vital y en el acompañamiento educativo familiar. }\end{array}$ \\
\hline & $\begin{array}{l}\text { Identificar y aplicar estrategias didácticas creativas e inclusivas ante } \\
\text { realidades diversas en población con problemas de salud. }\end{array}$ \\
\hline & $\begin{array}{l}\text { Saber seleccionar y aplicar herramientas digitales apropiadas para la } \\
\text { acción educativa y la comunicación. }\end{array}$ \\
\hline & $\begin{array}{l}\text { Diseñar y aplicar procesos evaluativos en programas y proyectos de } \\
\text { intervención e investigación en el campo de la pedagogía hospitalaria. }\end{array}$ \\
\hline & $\begin{array}{l}\text { Análisis y valoración de la realidad con la aplicación de metodologías } \\
\text { diversas. }\end{array}$ \\
\hline & Análisis de factores de riesgo y de protección vinculados al trabajo \\
\hline & Aplicar estrategias para el autocuidado de la salud \\
\hline \multirow{6}{*}{$\begin{array}{l}\text { Saber ser y } \\
\text { estar } \\
\text { (actitudes) }\end{array}$} & Responsabilidad \\
\hline & Empatía \\
\hline & Compromiso \\
\hline & Flexibilidad \\
\hline & Autoconfianza \\
\hline & Autocontrol \\
\hline
\end{tabular}

Fuente: Elaboración propia

A modo de ejemplo desarrollamos a continuación la competencia responsabilidad seguiendo el modelo de Rodríguez Moreno, Serreri y Cimmuto (2006) y Rodríguez Moreno, (2014). Estos autores atribuyen al concepto responsabilidad en el trabajo el significado de implicación como una demostración de la competencia personal y profesional. Este requisito es imprescindible en el ámbito de la educación hospitalaria, puesto que se trata de mejorar el bienestar de la persona con enfermedad y requiere una relación de ayuda y el establecimiento de vínculos afectivos. 
TABLA 2. Desarrollo de la competencia responsabilidad en la educación hospitalaria

\begin{tabular}{|c|c|}
\hline \multirow{2}{*}{\multicolumn{2}{|c|}{$\begin{array}{l}\text { El profesional de la educación hospitalaria demostrará en la práctica su responsabilidad } \\
\text { en el proceso educativo de niños, niñas y adolescentes con enfermedad. } \\
\text { 2. Definir el contexto curricular o laboral en el que deberá ser implementada }\end{array}$}} \\
\hline & \\
\hline \multicolumn{2}{|c|}{ Docente en aula y/o escuela hospitalaria } \\
\hline \multicolumn{2}{|c|}{ 3. Explicar el significado o sentido de la competencia } \\
\hline \multicolumn{2}{|c|}{$\begin{array}{l}\text { La responsabilidad es una competencia fundamental inherente a la propia } \\
\text { profesión educativa y de ayuda (helping profesión). Representa la implicación y el } \\
\text { compromiso con la confianza, el bienestar y la calidad de vida de la infancia y la } \\
\text { adolescencia en situación de vulnerabilidad la de sus familias. Comporta partir del } \\
\text { principio de que la educación es un derecho. }\end{array}$} \\
\hline $\begin{array}{l}\text { 4. Anotar los elementos } \\
\text { o componentes e la } \\
\text { competencia: } \\
\text { capacidades }\end{array}$ & $\begin{array}{l}\text { 5. Anotar las destrezas que convergen en la } \\
\text { capacidad }\end{array}$ \\
\hline $\begin{array}{l}\text { Conocer la carga física y } \\
\text { mental que significa ser } \\
\text { docente en un hospital. }\end{array}$ & $\begin{array}{l}\text { Saber cómo hay que cumplir con los compromisos } \\
\text { adquiridos con la institución. } \\
\text { Saber identificar los factores de riesgo por exceso de carga } \\
\text { de trabajo y sus consecuencias } \\
\text { Saber identificar la sobrecarga o el exceso de } \\
\text { responsabilidades auto atribuidas. } \\
\text { Saber cómo conciliar la carga laboral con la familiar. }\end{array}$ \\
\hline $\begin{array}{l}\text { Conocer las normativas } \\
\text { curriculares que regulan el } \\
\text { trabajo docente y las que } \\
\text { regulan el contexto } \\
\text { sanitario en relación con la } \\
\text { higiene y seguridad. }\end{array}$ & $\begin{array}{l}\text { Saber cumplir las normativas educativas y sanitarias. } \\
\text { Saber atender la diversidad. } \\
\text { Saber mantener la higiene personal y de los materiales } \\
\text { para la docencia. } \\
\text { Saber aplicar las normas de seguridad del hospital. }\end{array}$ \\
\hline $\begin{array}{l}\text { Implicarse en el trabajo } \\
\text { docente con los niños, niñas, } \\
\text { adolescentes hospitalizados y } \\
\text { sus familias. }\end{array}$ & $\begin{array}{l}\text { Actuar sin prejuicios. } \\
\text { Identificar criterios de eficiencia (evidencia y buenas } \\
\text { prácticas) y de calidad educativa. } \\
\text { Preocuparse por los resultados de aprendizaje y por el } \\
\text { estado emocional. } \\
\text { Atender a las expectativas e intereses de las personas } \\
\text { atendidas. } \\
\text { Cumplir con los principios éticos. }\end{array}$ \\
\hline $\begin{array}{l}\text { Implicarse en el trabajo } \\
\text { colaborativo con el resto de } \\
\text { los profesionales. }\end{array}$ & $\begin{array}{l}\text { Establecer relaciones interprofesionales de respeto. } \\
\text { Interesarse por el trabajo de otros profesionales } \\
\text { Informar de forma trasparente. } \\
\text { Aceptar las aportaciones de otros. }\end{array}$ \\
\hline
\end{tabular}

Fuente: elaboración propia

\section{La formación del docente hospitalario. Aprender a enseñar y a educar}

El docente hospitalario necesita una formación desde la transdisciplinariedad, donde se articulan los conocimientos de las ciencias de la educación con las ciencias de la salud, las ciencias sociales y humanas. 
Dado el contexto de vulnerabilidad por motivos de salud, coincidimos con Ortiz (1999), en que la formación debe tener un carácter humano y académico a la vez. Deberá incluir contenidos humanistas, que fomenten un pensamiento crítico y reflexivo y favorezcan una aproximación a la realidad empática, afectiva y mediada por valores éticos. Tal como afirman Sánchez Andrade y Pérez Padrón (2017), que vaya más allá de los contenidos disciplinares, para favorecer el desarrollo pleno como personas.

Combiene destacar la importancia de enfocar la formación del docente hospitalario mediante procesos de enseñanza y aprendizaje desde una perspectiva del aprendizaje basado en el trabajo, donde se integre la formación teórico-práctica, la experiencial y la práctica en los propios contextos de intervención. La interacción directa con profesionales y con la población destinataria, favorece el desarrollo de las competencias profesionales de forma más efectiva. Uno de los elementos más significativos de este proceso es el desarrollo de la capacidad reflexiva que incidirá tanto en el proceso de aprendizaje como en la práctica profesional (RODRÍGUEZ MORENO, 2006).

Coincidiendo con Ruiz Revert y García Raga (2019) la formación en pedagogía hospitalaria debe recibirla, además del y la docente hospitalaria, el o la docente de la escuela regular y todo profesional de la educación, que pueda tener en su grupo educativo un niño, niña o adolescente con enfermedad. Sin embargo para el contexto hospitalario deberá recibir una formación específica por la singularidad del entorno (sanitario versus educativo). En base a su investigación, Arreola (2019) refiere distintos elementos diferenciadores entre la escuela hospitalaria y la ordinaria que tiene que atender el profesional docente y, por tanto, deben tenerse en cuenta en la formación de estos profesionales: el clima socioemocional que se da en el alumnado; en el hospital está presente el dolor, el sufrimiento y el miedo; la diversidad por enfermedad, además de los distintos niveles educativos; dificultades para disponer de estrategias adaptadas a cada etapa educativa, a la situación de cada 
momento y suficientemente flexibles; necesidad de conocimientos sobre aspectos sanitarios además de los educativos.

Se plantean tres modalidades formativas en pedagogía hospitalaria:

\section{- Formación inicial universitaria:}

La formación que prepara y acredita para ejercer como profesional de la educación en cualquiera de sus modalidades (docente en educación infantil, primaria y secundaria, educación social, pedagogía y psicopedagogía) debería introducir este ámbito de intervención. Estas profesiones están implicadas, en la pedagogía hospitalaria, ya sea en los servicios educativos regulares, socioeducativos o del hospital, dado que todo niño, niña o adolescente en cualquiera de los espacios educativos donde se encuentre, tiene derecho a una educación inclusiva. Por otro lado, esta formación inicial contribuirá a reducir el desconocimiento social de esta disciplina ya que, según Ruiz Revert y García Raga (2019), también es una realidad poco conocida por los profesionales de la educación.

El estudio de estas autoras en el territorio español concluye que en los grados de magisterio del contexto español, ninguna universidad pública ofrece asignaturas relacionadas con la pedagogía hospitalaria. En relación con las universidades privadas, solamente la Universidad de Navarra imparte una asignatura con el título de pedagogía hospitalaria en los grados de Educación Infantil y Primaria (0-6 y 6 a 12 años respectivamente). Algunas universidades han ofertado contenidos o asignaturas con esta temática en especialidades o menciones: educación especial (Universidad de Vigo). En el grado de Pedagogía, según el estudio de Ruiz Revert y García Raga (2019), la Universidad de Burgos (obligatoria), la Universidad de Santiago de Comportela (optativa) y la Universidad de Navarra (optativa) ofrecen asignaturas relacionadas. En el grado de educación social, únicamente mencionan la Universitat de València, que ofrece una asignatura de Educación para la salud. 
La Facultad de Educación de la Universidad de Barcelona (UB) ${ }^{3}$, ofrece asignaturas en los grados que relacionan la educación y la salud o que incluyen contenidos relacionados con esta disciplina: en Educación Infantil se ofrece la asignatura obligatoria: "Infancia, salud y educación". En la mención de Atención a la Diversidad en Educación infantil y Primaria se contempla la pedagogía hospitalaria como uno de los ámbitos de diversidad. En los grados de Pedagogía y Educación Social se comparte la asignatura "Educación, Formación y Salud", que contiene un bloque destinado a la pedagogía hospitalaria. Hay que considerar también la importancia de la formación desde las prácticas externas curriculares en aulas hospitalarias, en fundaciones y asociaciones y otros organismos de salud, así como los Trabajos Finales de Grado que se refieren a este ámbito de actuación.

- Formación especializada universitaria:

La UB ofrece desde el curso 2015-2016 la titulación de Máster en pedagogía hospitalaria a lo largo de la vida, como máximo nivel académico en cuanto a la especialización, que ha iniciado su sexta edición el curso 20202021 y que cuenta con estudiantes de España y Latinoamérica, dado que se imparte en modalidad mixta (b-learning), mediante presencialidad virtual. Otras especialidades impartidas son los Diplomas de Especialización /Posgrados en pedagogía hospitalaria, así como cursos de expertos.

Los programas de doctorado de educación de las universidades permiten la realización de tesis doctorales sobre líneas de investigación relacionadas con la pedagogía hospitalaria. Representa una oportunidad de investigar y de este modo se contribuye a la construcción de conocimiento en este campo disciplinar.

\section{- Formación continuada:}

Si entendemos la educación desde una perspectiva permanente y a lo largo de la vida (lifelong learning), el desarrollo profesional implica la actualización y profundización de saberes, así como la reflexión de la

\footnotetext{
${ }^{3}$ Se pueden consultar los planes de estudios de Grados y Postgrados de la Facultad de Educación de la Universidad de Barcelona en el web: https://www.ub.edu/portal/web/educacio/.
} 
propia práctica. Para ello, la universidad, las organizaciones contratadoras y la administración pública deben asumir la coresponsabilidad en la formación continuada de los y las profesionales para garantizar buenas prácticas, especialmente cuando se trata de atender personas en situación de vulnerabilidad.

Un elemento clave en la formación es el desarrollo de la investigación colaborativa y participativa, cuyos resultados contribuirán a la construcción del conocimiento necesario para la formación y para la práctica profesional basada en evidencias.

\section{Conclusiones}

La educación en los hospitales es la práctica más extendida dirigida a los pacientes pediátricos en edad escolar que por motivos de salud no pueden acudir a un centro educativo regular. Su marco de actuación, la pedagogía hospitalaria, tiene sentido por el reconocimiento internacional del derecho a la educación y a la salud, entendiendo ésta desde una perspectiva biopsicosocial. Sin embargo, las instancias internacionales de prestigio en educación como la UNESCO demandan una educación de calidad para todos, es decir basada en los principios de equidad y de inclusión.

Estos principios no siempre se aplican de forma real y quedan reducidos a su expresión teórica y normativa. La equidad es uno de los objetivos principales en políticas educativas, sanitarias y sociales desde hace muchos años, aunque se avanza muy lentamente hacia su consecución.

La pedagogía hospitalaria representa una oportunidad para progresar en esa dirección. Observando su evolución, se abren nuevas esperanzas en la mejora hacia la calidad y hacia una cobertura cada vez más amplia de la población infantil y adolescente en edad escolar. Siguiendo la perspectiva de la educación a lo largo de la vida (Lifelong Learning), va ampliando su marco de actuación, que ya se hace visible en algunas experiencias y proyectos desarrollados en esta dirección. 
Resulta de especial relevancia situar la pedagogía hospitalaria desde una perspectiva amplia que incorpore estos enfoques y que la caracterice como una disciplina puesto que, mediante la investigación, dispone de un cuerpo de conocimientos basados en las evidencias y en las buenas prácticas. Esta dimensión le otorgará sentido y razón de ser, para el reconocimiento social como una profesión necesaria que contribuye a la educación de calidad.

Por otro lado, se plantea la necesidad de que los diferentes contextos de actuación de la pedagogía hospitalaria formen parte de un sistema educativo inclusivo de calidad en coordinación con la escuela regular, junto a la corresponsabilidad de la comunidad y de la administración pública.

Un eje fundamental para esta educación de calidad dirigida a personas con problemas de salud y sus familias, es el y la profesional. Es obvio que debe ser competente para desarrollar sus funciones de forma efectiva y ética. Como disciplina, la pedagogía hospitalaria también debe avanzar hacia la profesionalidad definiendo la especificidad de la profesión y promoviendo el desarrollo profesional, mediante una formación de calidad basada en las competencias generales de cualquier acto educativo y las específicas que corresponden a las diversas funciones que realiza.

La administración pública, las organizaciones contratadoras y los propios profesionales deben asumir la responsabilidad compartida de asegurar una formación inicial, especializada y continuada, mediante metodologías de enseñanza y aprendizaje que fomenten la capacidad reflexiva sobre la propia práctica y desde un enfoque basado en el trabajo que integre la formación teórico-práctica, la experiencial y la práctica en los propios contextos de intervención.

El camino está iniciado pero queda aún mucho por transitar. En este recorrido, aplicando las estrategias adecuadas, podemos ir alcanzando pequeñas metas que nos irán acercando cada vez más hacia una educación de calidad equitativa e inclusiva para todas las personas con problemas de salud y sus familias, revirtiendo a su vez en una mejora del bienestar y la calidad de vida. 


\section{Referencias}

\section{AGENCIA DE CALIDAD Y EVALUACIÓN SANITARIAS DE}

CATALUNYA. Atención integrada: ¿cuál es la idea clave?, 2016. Disponible en: https://blog.aquas.cat/2016/06/09/icic16-atencion-integrada/?lang=es. Acceso en: 31 ene 2021.

AINSCOW, M. Promoting inclusion and equity in education: lessons from international experiences, NordSTEP, v.6, p.7-16, marzo 2020. DOI: https://doi.org/10.1080/20020317.2020.1729587

ALBERTONI, L.; CHIARI, B. M. A inclusão escolar de alunos com doenças crônicas: professores e gestores dizem que.... Curitiba (Brasil): Appris, 2014.

ARREOLA, R. del C. Apuntes para la formación: la pedagogia hospitalaria, uma visión transdisciplinar. En: LIRA, L. (coord..). Experiencias de investigación multidisciplinar. Aprendizaje integrado: investigación, conocimiento y práctica. Universidad Marista de Guadalajara (España), 2019, p. 387-414.

BOZU, Z. El perfil de las competencias profesionales del profesorado de la ESO. PREAL, 2007. Disponible en: https://nanopdf.com/download/el-perfilde-las-competencias-profesionales-del-profesorado-de_pdf\# . Acceso en: 31 ene 2021.

CASTILlO, M. A. Aula hospitalaria. Calidad de vida. Ceuta: Archivo General de Ceuta, 2015

COMITÉ DE GESTIÓN DE TUNING. Una introducción a Tuning Educational Structures in Europe. La contribución de las universidades al proceso de Bolonia. Universidad de Deusto, 2006. Disponible en: https://www.unideusto.org/tuningeu/images/stories/documents/General_Broc hure_Spanish_version.pdf. Acceso en: 31 ene 2021.

GENERALITAT DE CATALUNYA. Marc d'actuació de les aules hospitalàries. Departament d'Educació. Generalitat de Catalunya, 2007. Disponible en: http://xtec.gencat.cat/web/.content/alfresco/download/Company_Home/Centr es/xtec_public/Marc_actuacio_AH_1.pdf. Acceso en: 31 ene 2021.

GONZÁLEZ-SIMANCAS, J. L.; POLAINO-LORENTE, A. Pedagogía Hopitalaria. Actividad educativa en ambientes clínicos. Madrid: Narcea, 1990. 
GRAU, C.; ORTIZ, C. La pedagogia hospitalaria em el marco de uma educación inclusiva. Málaga: Ediciones Aljibe, 2001.

IMBERNÓN, F.; CANTO, P. J. La formación y el desarrollo profesional del profesorado en España y Latinoamérica. Sinéctica, v. 41, p. 1-12, diciembre 2013. Disponible en: https://sinectica.iteso.mx/index.php/SINECTICA/article/view/36. Acceso en: 31 ene 2021.

LIZASOÁIN, O. Pedagogía hospitalaria. Guía para la atención psicoeducativa del alumno enfermo. Madrid: Editorial Síntesis, 2016.

MOLINA, M.C.; BORI, M. Ámbitos de intervención en pedagogía hospitalaria. En: Bobadilla, A., Bori, M., Cardone, P., Ferreira, M., Lizásoain, O., Molina, M. C., Riquelme, S., Saruwatari, G., Simoes, E. y Violant, V. La Pedagogía Hospitalaria Hoy. Análisis de las Políticas, los ámbitos y la formación de profesionales. Santiago de Chile: Santillana, 2013, p. 70-107.

MOLINA, M.C. Pedagogía hospitalaria. Claves teóricas y enfoques para la práctica. Barcelona: Octaedro editorial, 2020.

OJEDA, M. C. Rasgos de la identidad del profesor de enseñanza media en su trayectoria de formación y desempeño profesionales. ¿Cómo, cuándo y con quiénes adquiere su condición de profesor? REDIE, v. 10, n. 2, p. 1-14, noviembre 2008. Disponible en: http://redie.uabc.mx/vol10no2/contenidoojeda.html. Acceso en: 31 ene 2021.

OLIVEIRA, H. H. La construcción de la profesionalidad docente. Educación, vol. XXII, n. 42, p. 97-115, marzo 2013, Disponible en: https://dialnet.unirioja.es/servlet/articulo?codigo=5056907. Acceso en: 31 ene 2021.

ORTIZ, C. Formación de los profesionales del contexto hospitalario. Profesorado. Revista de curriculum y formación del profesorado, v. 3, n. 2, p. 105-120, 1999. Disponible en: https://www.ugr.es/ recfpro/rev32ART5.pdf. Acceso en: 31 ene 2021.

RODRÍGUEZ MORENO, M.L. Evaluación, balance y formación de competencias laborales transversales. Propuestas para mejorar la calidad en la formación profesional y en el mundo del trabajo. Barcelona: Laertes educación, 2006.

RODRÍGUEZ MORENO, M.L. La pasión por aprender a aprender.

Desarrollo de la competencia estratégica. Guía didáctica para la universidad y la empresa. Barcelona: Laertes educación, 2014.

RODRÍGUEZ MORENO, M.L.; SERRERI, P.; DEL CIMMUTO, A. Desarrollo de competencias: teoría y práctica. Barcelona: Laertes educación, 2010. 
RUIZ REVERT, M.; GARCÍA RAGA, L. Pedagogía hospitalaria. Una asignatura pendiente en la formación universitaria de los profesionales de la educación. Edetania: estudios y propuestas socio-educativas, n.. 55, 2019, p. 181-202. Disponible en: https://dialnet.unirioja.es/servlet/articulo?codigo=7054406. Acceso en: 31 ene 2021.

SACRISTÁN, J. G. Consciência e ação sobre a prática como libertação profissional dos professores. En Nóvoa, A. (comp.). Profissão professor. (Coleção Ciências da Educação). Porto, Portugal: Ed. Porto, 1991, p. 63-92.

SÁNCHEZ ANDRADE, V.; PÉREZ PADRÓN, M.C. La formación humanista: un encargo para la educación. Revista Universidad y Sociedad, v. 9, n. 3, p. 265-269, 2017. Disponible en:

http://scielo.sld.cu/scielo.php?script=sci arttext\&pid=S221836202017000300041\&lng=es\&tlng=es. Acceso en: 31 ene 2021.

TEJADA, J. Profesionalidad docente. En: DE LA TORRE, S.; BARRIOS, O. (coords.). Estrategias didácticas innovadoras, p. 62-76. Barcelona: Octaedro, 2002.

UNIVERSITAT DE BARCELONA. Competències transversals de la Universitat de Barcelona. Universitat de Barcelona, 2008. Disponible en: http://diposit.ub.edu/dspace/bitstream/2445/2941/1/comp_trans_UB.pdf. Acceso en: 31 ene 2021. 